Journal of Applied ANALysis

Vol. 7, No. 2 (2001), pp. 209-224

\title{
THE NEVANLINNA THEOREM OF THE CLASSICAL THEORY OF MOMENTS REVISITED
}

\author{
M. URREA, I. M. TKACHENKO and P. FERNÁNDEZ DE CÓRDOBA \\ Received March 31, 1999 and, in revised form, March 16, 2001
}

\begin{abstract}
The canonical solutions of the truncated Hamburger moment problem (both in the classical and degenerate cases) are found. The Nevanlinna theorem which provides the noncanonical solutions of the truncated Hamburger problem is also rederived in the framework of the operator approach.
\end{abstract}

\section{Introduction}

Over a century ago Stieltjes published a work [13] which initiated the development of the theory of moments. Stieltjes even managed to solve the problem of finding a nondecreasing function of bounded variation, $\sigma(t), t \in$ $[0,+\infty)$ with given moments $\mu_{k}=\int_{0}^{+\infty} t^{k} d \sigma(t), k=0,1,2, \ldots$ connecting

2000 Mathematics Subject Classification. Primary 30E05, 30E10; Secondary 82C70, $82 \mathrm{D} 10$.

Key words and phrases. Power moments, Nevanlinna's theorem, operator approach.

The work was partially financed by the Institució Valenciana d'Estudis i Investigació (I.V.E.I.) (grant No. 024-94). The financial support of the Polytechnic University of Valencia is also acknowledged.

ISSN 1425-6908 C Heldermann Verlag. 
certain continued fractions with the Cauchy-type integral

$$
\int_{0}^{\infty} \frac{d \sigma(t)}{t+z}
$$

Though many results on the moment problem perhaps look now like "folklore", it is worth-while to reformulate or rederive some of them especially those for the truncated problems where only a finite set of moments is given [8], [9] . Such rederivation from the position of modern applications appears to be fruitful for the trigonometric problem of moments (i.e. for the Carathéodory-class functions) application to digital signal processing and for some new topics in the operator theory or the probability theory (see for example [6] and references therein). Recently Curto and Fialkow ([4]) using a fine linear algebra technique to describe a recursive model for singular positive Hankel matrices have pointed out new elements even in such a guide-book tale as necessary and sufficient conditions for existence or uniqueness of positive Borel or finitely atomic measures which solve the truncated, including degenerate problems (i.e. problems with the singular Hankel matrix of moments) of Hamburger, Hausdorff and Stieltjes.

Here, we are interested in the problem of moments for the Nevanlinnaclass functions $\mathcal{N}$ (also named the Riesz-class functions, i.e. the class of analytical functions transforming the set $G_{z}=\{z \in \mathbb{C}: \operatorname{Im}(z)>0\}$ into $G_{\omega}=\{\omega \in \mathbb{C}: \operatorname{Im}(\omega) \geq 0\}$, realized by the Riesz-Herglotz transformation), having in mind applications to the theory of orthogonal polynomials, the operator theory, ... even in the context of strongly coupled plasmas $[1,11]$.

The canonical solutions of the Hamburger moment problem [5] (both in the classical and degenerate cases) are found. The Nevanlinna theorem which provides the noncanonical solutions of the Hamburger problem is also rederived using the operator approach elaborated and briefly outlined for the moment problem in the renowned paper by M.G. Krein [7].

\section{Canonical solutions of the truncated Hamburger problem}

The Hamburger problem of the theory of moments $[2,9,10,12]$ is formulated in the following way:

Given a set of real numbers $\left\{\mu_{0}, \mu_{1}, \mu_{2}, \ldots, \mu_{2 n}\right\}, n=0,1,2, \ldots$, to find all distributions $\sigma(t)$ such that

$$
\int_{-\infty}^{\infty} t^{k} d \sigma(t)=\mu_{k}, \quad k=0,1,2, \ldots, 2 n .
$$


Theorem 2.1. A system of real numbers $\left\{\mu_{0}, \mu_{1}, \mu_{2}, \ldots, \mu_{2 n}\right\}, n=0,1,2$, $\ldots$, admits the representation of equation (2.1) if and only if

1. The Hankel matrix $\Lambda_{n}=\left[\mu_{k+j}\right]_{k, j=0}^{n}$ is nonnegative;

2. For each vector $\boldsymbol{\xi}=\left(\xi_{j}\right)_{j=0}^{n} \in \mathbb{C}^{n+1}$ with the component $\xi_{n}=0$, the equalities

$$
\left(\Lambda_{n} \boldsymbol{\xi}\right)_{j}=\sum_{k=0}^{n-1} \mu_{j+k} \xi_{k}=0, \quad j=0,1, \ldots, n
$$

imply

$$
\sum_{k=0}^{n-1} \mu_{n+1+k} \xi_{k}=0 .
$$

If in addition $\Lambda_{n} \geq 0$ is a singular matrix, then there is only one distribution which satisfies (2.1), and it is supported by a set of $s \leq n$ points.

Proof. Suppose that relations (2.1) hold.

For an arbitrary set $\left\{\xi_{0}, \xi_{1}, \xi_{2}, \ldots, \xi_{n}\right\}$ of complex numbers define

$$
P(t)=\xi_{0}+\xi_{1} t+\xi_{2} t^{2}+\ldots+\xi_{n} t^{n},
$$

then it follows from $(2.1)$ that

$$
\left(\Lambda_{n} \boldsymbol{\xi}, \boldsymbol{\xi}\right) \equiv \sum_{k, j=0}^{n} \mu_{k+j} \xi_{k} \overline{\xi_{j}}=\int_{-\infty}^{\infty}|P(t)|^{2} d \sigma(t) \geq 0 .
$$

Hence the Hankel matrix $\Lambda_{n}=\left[\mu_{k+j}\right]_{k, j=0}^{n}$ is nonnegative.

If the Hankel matrix $\Lambda_{n}$ is non-negative and

$$
\left(\Lambda_{n} \boldsymbol{\xi}, \boldsymbol{\xi}\right)=\int_{-\infty}^{\infty}|P(t)|^{2} d \sigma(t)=0
$$

for the non-zero vector $\boldsymbol{\xi} \in \mathbb{C}^{n+1}$ then the support of the distribution $d \sigma(t)$ belongs to the set $\left\{\alpha_{j}\right\}_{j=0}^{s}, s \leq n$ of real roots of the polynomial $P(t)$. Furthermore, it is evident that for vectors $\zeta_{j}$ to which correspond the polynomials

$$
P_{j}(t)=\frac{\prod_{m \neq j}^{s}\left(t-\alpha_{m}\right)}{\prod_{m \neq j}^{s}\left(\alpha_{j}-\alpha_{m}\right)},
$$


we have

$$
\left(\Lambda_{n} \boldsymbol{\zeta}_{j}, \boldsymbol{\zeta}_{j}\right)=\int_{-\infty}^{\infty}\left|P_{j}(t)\right|^{2} d \sigma(t)=\sigma\left(\alpha_{j}+0\right)-\sigma\left(\alpha_{j}-0\right)
$$

Thus, since $P(t)$, and consequently $\left\{\alpha_{j}\right\}_{j=0}^{s}$, depend only and exclusively on the given moments $\left\{\mu_{k}\right\}_{k=0}^{2 n}$, we conclude that only one distribution $d \sigma(t)$ can exist to satisfy (2.1), if $\Lambda_{n} \geq 0$ and (2.5) holds for some $\Lambda_{n} \neq 0$, and in addition this unique distribution is supported by $s \leq n$ points.

Recall that for any matrix (operator) $\Lambda_{n} \geq 0$ the equality $\left(\Lambda_{n} \boldsymbol{\xi}, \boldsymbol{\xi}\right)=0$ holds if and only if $\Lambda_{n} \boldsymbol{\xi}=0$. For a vector $\boldsymbol{\xi} \in \mathbb{C}^{n+1}$ with the coordinate $\xi_{n}=0$ the equalities $(2.2)$ mean that $\Lambda_{n} \boldsymbol{\xi}=0$. Taking a vector $\boldsymbol{\eta} \in \mathbb{C}^{n+1}$ with $\eta_{0}=0 ; \eta_{j}=\xi_{j-1}, j=1, \ldots, n$, we see that $P(t)=t P(t)$, so that we have:

$$
\left(\Lambda_{n} \boldsymbol{\eta}, \boldsymbol{\eta}\right)=\int_{-\infty}^{\infty}|P(t)|^{2} d \sigma(t)=\int_{-\infty}^{\infty} t^{2}|P(t)|^{2} d \sigma(t) .
$$

But the equality (2.5) for $\boldsymbol{\xi}$ forces

$$
\int_{-\infty}^{\infty} t^{2}|P(t)|^{2} d \sigma(t)=0
$$

Hence $\left(\Lambda_{n} \boldsymbol{\eta}, \boldsymbol{\eta}\right)=0$ and, as follows, $\Lambda_{n} \boldsymbol{\eta}=0$. In particular $\left(\Lambda_{n} \boldsymbol{\xi}\right)_{n}=0$, i.e. (2.3) holds. To prove the sufficiency:

1. Assume first that the matrix $\left[\mu_{k+j}\right]_{k, j=0}^{n}$ is positive, i.e.,

$$
\sum_{k, j=0}^{n} \mu_{k+j} \xi_{k} \overline{\xi_{j}}>0
$$

for any vector $\boldsymbol{\xi}$ such that $\sum_{k=0}^{n}\left|\xi_{k}\right|^{2}>0$.

Construct a $(n+1)$-dimensional Hilbert space $\mathcal{H}$ of vectors $\boldsymbol{\xi}=\left(\xi_{0}, \xi_{1}\right.$, $\left.\xi_{2}, \ldots, \xi_{n}\right)$ taking the Hermitian form

$$
\langle\boldsymbol{\xi}, \boldsymbol{\eta}\rangle=\sum_{k, j=0}^{n} \mu_{k+j} \xi_{k} \overline{\eta_{j}}
$$

as a scalar product.

Denote by $\left\{\mathbf{h}_{0}, \mathbf{h}_{1}, \ldots, \mathbf{h}_{n}\right\}$ an orthonormal basis of the space $\mathcal{H}$ obtained from the canonical basis $\left\{\mathbf{e}_{0}, \mathbf{e}_{1}, \ldots, \mathbf{e}_{n}\right\}$ by the Gram-Schmidt procedure. Introduce a Hermitian operator $\mathrm{A}_{0}$ in $\mathcal{H}$ such that

$$
\mathrm{A}_{0} \mathbf{e}_{k}=\mathbf{e}_{k+1}, k=0,1, \ldots, n-1 .
$$


Since

$$
\left\langle\mathbf{e}_{k+1}, \mathbf{e}_{j}\right\rangle=\mu_{k+j+1}=\left\langle\mathbf{e}_{k}, \mathbf{e}_{j+1}\right\rangle
$$

then for any two vectors $\boldsymbol{\xi}, \boldsymbol{\eta} \in \operatorname{span}\left(\mathbf{e}_{0}, \mathbf{e}_{1}, \ldots, \mathbf{e}_{n-1}\right)$

$$
\left\langle\mathrm{A}_{0} \boldsymbol{\xi}, \boldsymbol{\eta}\right\rangle=\left\langle\boldsymbol{\xi}, \mathrm{A}_{0} \boldsymbol{\eta}\right\rangle \text {. }
$$

Consider now the vectors $\mathrm{A}_{0} \mathbf{h}_{j}$ with $0 \leq j<n$. By definition $\mathbf{h}_{j}$ is orthogonal to all vectors $\mathbf{h}_{i}$ with $i<j$, and is a linear combination of the vectors $\mathbf{e}_{l}$ with $l \leq j$. Hence, the vector $\mathrm{A}_{0} \mathbf{h}_{j}$ will be orthogonal to all vectors $\left\{\mathbf{h}_{j+2}, \ldots, \mathbf{h}_{n}\right\}$, and due to the hermiticity condition (2.10), $\mathrm{A}_{0} \mathbf{h}_{j}$ is also orthogonal to all vectors $\left\{\mathbf{h}_{0}, \ldots, \mathbf{h}_{j-2}\right\}$. Setting $\overline{b_{-1}}=0$ we see that

$$
\mathrm{A}_{0} \mathbf{h}_{j}=b_{j} \mathbf{h}_{j+1}+a_{j} \mathbf{h}_{j}+\overline{b_{j-1}} \mathbf{h}_{j-1}, 0 \leq j \leq n-1 .
$$

Observe that the coefficients $a_{j}$ in (2.11) are real.

Let us now extend $\mathrm{A}_{0}$ to an operator $\mathrm{A}$ on the whole Hilbert space $\mathcal{H}$ by relations:

$$
A \mathbf{h}_{j}= \begin{cases}b_{j} \mathbf{h}_{j+1}+a_{j} \mathbf{h}_{j}+\overline{b_{j-1}} \mathbf{h}_{j-1} & \text { if } 0 \leq j \leq n-1, \\ a_{n} \mathbf{h}_{n}+b_{n-1} \mathbf{h}_{n-1} & \text { if } j=n\end{cases}
$$

where $a_{n}$ is an arbitrary real number.

Notice that for the orthonormal basis $\left\{\mathbf{h}_{0}, \ldots, \mathbf{h}_{n}\right\}$ the matrix of operator $\mathrm{A}$ is Hermitian. Therefore $\mathrm{A}$ is a self-adjoint operator and there exists a resolution of the identity $\mathcal{E}_{t}$ such that for any $\boldsymbol{\xi}, \boldsymbol{\eta} \in \mathcal{H}$

$$
\langle\mathrm{A} \boldsymbol{\xi}, \boldsymbol{\eta}\rangle=\int_{-\infty}^{\infty} t\left\langle d \mathcal{E}_{t} \boldsymbol{\xi}, \boldsymbol{\eta}\right\rangle
$$

Consider now the following non-decreasing function

$$
\sigma(t)=\left\langle\mathcal{E}_{t} \mathbf{e}_{0}, \mathbf{e}_{0}\right\rangle, t \in \mathbb{R},
$$

of pure jumps. Since by construction

$$
\mathrm{A}^{k} \mathbf{e}_{0}=\mathbf{e}_{k}, \quad k=0,1, \ldots, n,
$$

and

$$
\begin{aligned}
\mu_{j+k} & =\left\langle\mathbf{e}_{j}, \mathbf{e}_{k}\right\rangle=\left\langle\mathrm{A}^{j} \mathbf{e}_{0}, \mathrm{~A}^{k} \mathbf{e}_{0}\right\rangle \\
& =\left\langle\mathrm{A}^{j+k} \mathbf{e}_{0}, \mathbf{e}_{0}\right\rangle=\int_{-\infty}^{\infty} t^{j+k} d \sigma(t) .
\end{aligned}
$$

for any $j, k=0,1, \ldots, n$ we see that $\mu_{j}$ are the moments of the distribution $d \sigma(t)$. All distributions $d \sigma(t)$ constructed in such a way will be called canonical. 
2. Suppose now that there exist vectors $\boldsymbol{\xi}=\left(\xi_{k}\right)_{k=0}^{n} \in \mathbb{C}^{n+1}$ such that

$$
\sum_{k=0}^{n}\left|\xi_{k}\right|^{2}>0
$$

and

$$
\sum_{j, k=0}^{n} \mu_{k+j} \xi_{k} \overline{\xi_{j}}=0 .
$$

The vectors $\boldsymbol{\xi}$ satisfying (2.16) form a linear subspace $\mathcal{L} \subset \mathbb{C}^{n+1}$. Let us consider the factor space $\mathcal{F}=\mathbb{C}^{n+1} \backslash \mathcal{L}$ as a Hilbert space, where the scalar product of the classes $\tilde{\boldsymbol{\xi}}, \tilde{\boldsymbol{\eta}}$ ( generated by the vectors $\boldsymbol{\xi}, \boldsymbol{\eta} \in \mathbb{C}^{n+1}$ ) will be represented as $\langle\langle\tilde{\boldsymbol{\xi}}, \tilde{\boldsymbol{\eta}}\rangle\rangle$, and will be defined as the scalar product of their corresponding vectors in the degenerate Hilbert space $\mathcal{H}$, i.e., $\langle\boldsymbol{\xi}, \boldsymbol{\eta}\rangle$.

Let $\tilde{\mathbf{e}}_{0}, \tilde{\mathbf{e}}_{1}, \ldots, \tilde{\mathbf{e}}_{n} \in \mathcal{F}$ be the classes corresponding to $\mathbf{e}_{0}, \mathbf{e}_{1}, \ldots, \mathbf{e}_{n}$. Note that

$$
\tilde{\mathbf{e}}_{n} \in \operatorname{span}\left(\tilde{\mathbf{e}}_{0}, \ldots, \tilde{\mathbf{e}}_{n-1}\right) .
$$

In fact, since $\operatorname{dim} \mathcal{F} \leq n$ there is $\boldsymbol{\xi} \in \mathbb{C}^{n+1} \backslash\{0\}$ such that $\sum_{i=0}^{n} \xi_{i} \tilde{\mathbf{e}}_{i}=0$, i.e., $\boldsymbol{\xi} \in \mathcal{L}$ and the assertion follows if $\xi_{n} \neq 0$. If $\xi_{n}=0$, then $\boldsymbol{\eta}=$ $\left(0, \xi_{0}, \ldots, \xi_{n-1}\right) \in \mathcal{L} \backslash\{0\}$ by the assumption (2) of the theorem. If $\xi_{n-1}=0$ we similarly get $\left(0,0, \xi_{0}, \ldots, \xi_{n-2}\right) \in \mathcal{L} \backslash\{0\}$ and after finitely many steps we get $\left(0, \ldots, 0, \xi_{0}, \ldots, \xi_{n-p}\right) \in \mathcal{L} \backslash\{0\}$ with $\xi_{n-p} \neq 0$ but this shows that $(2.17)$ holds.

The relations

$$
\mathrm{B} \tilde{\mathbf{e}}_{k}=\tilde{\mathbf{e}}_{k+1}, k=0, \ldots, n-1,
$$

define a linear operator in $\mathcal{F}$ provided that

$$
\sum_{i=0}^{n-1} \xi_{i} \tilde{\mathbf{e}}_{i}=0 \quad \text { implies } \sum_{i=0}^{n-1} \xi_{i} \tilde{\mathbf{e}}_{i+1}=0 .
$$

But this follows from condition (2) of the theorem. By (2.17) and (2.18) B is uniquely defined also on the class $\tilde{\mathbf{e}}_{n}$. It is easy to see from (2.18) and the definition of the scalar product of $\mathcal{F}$ that $\mathrm{B}$ is self-adjoint. Thus there is the identity resolution $\tilde{\mathcal{E}}_{t}$ associated with B such that for each class $\tilde{\boldsymbol{\xi}} \in \mathcal{F}$ we have

$$
\mathrm{B} \tilde{\boldsymbol{\xi}}=\int_{-\infty}^{\infty} t d \tilde{\mathcal{E}}_{t} \tilde{\boldsymbol{\xi}}
$$

Taking now the nondecreasing function of bounded variation

$$
\tilde{\sigma}(t)=\left\langle\left\langle\tilde{\mathcal{E}}_{t} \tilde{\mathbf{e}}_{0}, \tilde{\mathbf{e}}_{0}\right\rangle\right\rangle, \quad t \in \mathbb{R},
$$


we conclude that

$$
\begin{aligned}
\mu_{j+k} & =\left\langle\left\langle\tilde{\mathbf{e}}_{j}, \tilde{\mathbf{e}}_{k}\right\rangle\right\rangle=\left\langle\left\langle\mathrm{B}^{j} \tilde{\mathbf{e}}_{0}, \mathrm{~B}^{k} \tilde{\mathbf{e}}_{0}\right\rangle\right\rangle \\
& =\left\langle\left\langle\mathrm{B}^{j+k} \tilde{\mathbf{e}}_{0}, \tilde{\mathbf{e}}_{0}\right\rangle\right\rangle=\int_{-\infty}^{\infty} t^{j+k} d \widetilde{\sigma}(t) .
\end{aligned}
$$

The solution constructed in this way is the unique solution of the truncated Hamburger moment problem (2.1). It will also be called canonical like in the non-degenerate case.

\section{Description of canonical solutions}

We now apply the above operator arguments to obtain the description formula for the canonical solutions.

For an arbitrary canonical distribution $\sigma(t)$ consider the function

$$
\Omega(z)=\int_{-\infty}^{\infty} \frac{d \sigma(t)}{t-z}
$$

also representable as:

$$
\Omega(z)=\left\langle(\mathrm{A}-z I)^{-1} \mathbf{e}_{0}, \mathbf{e}_{0}\right\rangle
$$

$I$ being the identity operator. Let us assume first that we deal with the non-degenerate case. Taking into account that A is a self-adjoint operator in an $(n+1)$-dimensional space and relation $(3.2)$ we see that $\Omega(z)$ is a rational function of the Nevanlinna class $\mathcal{N}$ and

$$
\lim _{z \rightarrow \infty} z^{2 n+1}\left\{\Omega(z)+\frac{\mu_{0}}{z}+\frac{\mu_{1}}{z^{2}}+\ldots+\frac{\mu_{2 n-1}}{z^{2 n}}\right\}=-\mu_{2 n},
$$

uniformly in any angle $\delta \leq \arg (z) \leq \pi-\delta, 0<\delta<\pi$. Notice that the set of these functions for all canonical distributions $d \sigma(t)$ is parametrized by the real numbers $a_{n}$ appearing in (2.12).

To obtain the corresponding description formula for all such $\Omega(z)$ let us find an appropriate explicit expression for the resolvent of operator A. To this end observe that since the vectors

$$
\mathrm{A}^{j} \mathbf{e}_{0}=\int_{-\infty}^{\infty} t^{j} d \mathcal{E}_{t} \mathbf{e}_{0}, j=0,1, \ldots, n
$$

form a basis of $\mathcal{H}$, any vector $\phi \in \mathcal{H}$ can be represented as

$$
\phi=\int_{-\infty}^{\infty} \phi(t) d \mathcal{E}_{t} \mathbf{e}_{0}
$$


where $\phi(t)$ is a polynomial of degree not higher than $n$. Thus, as usual, $\mathcal{H}$ can be realized as a Hilbert space of functions, the elements of which are polynomials of a degree not higher than $n$, the scalar product being defined as

$$
\langle\phi, \gamma\rangle=\int_{-\infty}^{\infty} \phi(t) \overline{\gamma(t)} d \sigma(t)
$$

$\gamma(t)$ being also a polynomial of degree not higher than $n$. For such realizations the vectors $\left\{\mathbf{h}_{0}, \ldots, \mathbf{h}_{n}\right\}$ transform into the system of orthogonal polynomials $\left\{h_{0}, \ldots, h_{n}(t)\right\}$ with respect to the canonical distribution $d \sigma(t)$. Emphasize that these polynomials are the same for all canonical distributions.

Recall that if $\operatorname{det} \Lambda_{n}>0$ then the orthogonal polynomials $h_{j}(z), j=$ $0,1, \ldots, n$, obtained by the Gram-Schmidt procedure are

$$
h_{n}(z)=\frac{1}{\sqrt{\Delta_{n-1} \Delta_{n}}} \operatorname{det}\left(\begin{array}{cccc}
\mu_{0} & \mu_{1} & \cdots & \mu_{n} \\
\mu_{1} & \mu_{2} & \cdots & \mu_{n+1} \\
\vdots & \vdots & \vdots & \vdots \\
\mu_{n-1} & \mu_{n} & \cdots & \mu_{2 n-1} \\
1 & z & \cdots & z^{n}
\end{array}\right), n=1,2, \ldots
$$

where

$$
\Delta_{j}=\operatorname{det}\left(\begin{array}{cccc}
\mu_{0} & \mu_{1} & \cdots & \mu_{j} \\
\mu_{1} & \mu_{2} & \cdots & \mu_{j+1} \\
\vdots & \vdots & \vdots & \vdots \\
\mu_{j} & \mu_{j+1} & \cdots & \mu_{2 j}
\end{array}\right) \quad, \quad j=0,1,2, \ldots
$$

while $\Delta_{-1}=1$ and $h_{0}(z)=\mu_{0}^{-1 / 2}$.

Consider for $z, \operatorname{Im} z>0$, and an arbitrary $\mathbf{f} \in \mathcal{H}$ the equation

$$
(\mathrm{A}-z I) \mathbf{g}=\mathbf{f} \text {. }
$$

Realizing $\mathbf{f}$ and $\mathbf{g}$ as polynomials $f(t), g(t)$ of degree not greater than $n$ and representing $g(t)$ as the orthogonal sum

$$
g(t)=g_{0}(t)+C h_{n}(t),
$$

where $g_{0}(t)$ is a polynomial of degree not greater than $n-1$ and $C$ is a complex number, we can with help of (2.12) write (3.6) as the equality

$$
(t-z) g_{0}(t)+C\left[\left(a_{n}-z\right) h_{n}(t)+b_{n-1} h_{n-1}(t)\right]=f(t) .
$$

For $t=z, \operatorname{Im} z>0,(3.7)$ gives

$$
C\left[\left(a_{n}-z\right) h_{n}(z)+b_{n-1} h_{n-1}(z)\right]=f(z) .
$$


Proposition 3.1. For any parameter $w$ with non-positive imaginary part the polynomial

$$
Q_{w}(t)=(w-t) h_{n}(t)+b_{n-1} h_{n-1}(t)
$$

has no zeros in the open upper half-plane.

Proof. Observe that the equality $Q_{w}(z)=0, \operatorname{Im} z>0$, would mean that the rational function

$$
g(t)=\frac{Q_{w}(t)}{t-z}
$$

actually is a polynomial of degree $n$. Let $\mathbf{g}$ be the corresponding vector and consider the vector $\mathbf{g}_{0}$, to which corresponds the non-zero polynomial

$$
g_{0}(t)=\frac{Q_{w}(t)}{t-z}+h_{n}(t)=\frac{(w-z) h_{n}(t)+b_{n-1} h_{n-1}(t)}{t-z},
$$

which is of degree not greater than $n-1$. Let the operator $\mathrm{A}_{w}$ be defined by formula (2.12) but with the parameter $w$ instead of $a_{n}$. The matrix of the operator $\mathrm{A}_{w}$ for the orthonormal basis $\left\{\mathbf{h}_{0}, \ldots, \mathbf{h}_{n}\right\}$ is either Hermitian (in the case $\operatorname{Im} w=0$ ) or it has non-positive imaginary part (in the case $\operatorname{Im} w<0)$. In both cases the operator $\mathbf{A}_{w}$ has no eigenvalues with positive imaginary parts. But from (3.9) we get immediately that the polynomial corresponding to the vector $\left(\mathrm{A}_{w}-z\right)$ g equals identically to zero, i.e. that

$$
\left(\mathrm{A}_{w}-z\right) \mathbf{g}=0
$$

a contradiction.

Returning to equalities (3.7), (3.8) with account of Proposition 3.1 for $\operatorname{Im} z>0$ we obtain

$$
C=\frac{f(z)}{\left(a_{n}-z\right) h_{n}(z)+b_{n-1} h_{n-1}(z)}
$$

and

$$
g(t)=\frac{f(t)}{t-z}-\frac{f(z)}{t-z} \frac{\left(a_{n}-z\right) h_{n}(t)+b_{n-1} h_{n-1}(t)}{\left(a_{n}-z\right) h_{n}(z)+b_{n-1} h_{n-1}(z)} .
$$

In particular, for the polynomial $u(t)$ corresponding to the vector $(\mathrm{A}-z I)^{-1} \mathbf{e}_{0}, e_{0}(t) \equiv 1$, we obtain

$$
\begin{aligned}
u(t) & = \\
& -\frac{\left(a_{n}-t\right)\left(h_{n}(t)-h_{n}(z)\right)+b_{n-1}\left(h_{n-1}(t)-h_{n-1}(z)\right)-(t-z) h_{n}(t)}{(t-z)\left[\left(a_{n}-z\right) h_{n}(z)+b_{n-1} h_{n-1}(z)\right]} .
\end{aligned}
$$


In order to determine the function $\Omega(z)$, let us introduce in parallel with $\left\{h_{0}, \ldots, h_{n}(t)\right\}$ the system of (second kind, or associate) polynomials:

$$
k_{j}(t)=\int_{-\infty}^{\infty}\left(h_{j}(t)-h_{j}(s)\right) \frac{d \sigma(s)}{t-s}, j=0,1, \ldots, n .
$$

Making use of (3.2), (3.5) and taking into account that

$$
\int_{-\infty}^{\infty} h_{n}(t) d \sigma(t)=0
$$

we get

$$
\begin{aligned}
\Omega_{a_{n}}(z) & =\int_{-\infty}^{\infty} \frac{d \sigma_{a_{n}}(t)}{t-z} \\
& =-\frac{\left(a_{n}-z\right) k_{n}(z)+b_{n-1} k_{n-1}(z)}{\left(a_{n}-z\right) h_{n}(z)+b_{n-1} h_{n-1}(z)}
\end{aligned}
$$

with $a_{n}$ being an arbitrary real number.

Further, if all the moments up to the $2(n+1)$-th were known and the corresponding Hankel matrix were positive, we could rewrite the last formula for $n \rightarrow n+1$ (one should amplify the sequence of moments keeping it positive) and introduce the polynomial $h_{n+1}(t)$ in a way that the following relation would be valid:

$$
t h_{n}(t)=b_{n} h_{n+1}(t)+\hat{a}_{n} h_{n}(t)+b_{n-1} h_{n-1}(t),
$$

where

$$
\widehat{a}_{n}=\int_{-\infty}^{\infty} t\left|h_{n}(t)\right|^{2} d \sigma(t)
$$

Then, instead of (3.14), one would get

$$
\Omega(z)=\int_{-\infty}^{\infty} \frac{d \sigma(t)}{t-z}=-\frac{k_{n+1}(z)+\nu k_{n}(z)}{h_{n+1}(z)+\nu h_{n}(z)}
$$

where

$$
\nu=\frac{\widehat{a}_{n}-a_{n}}{b_{n}}
$$

is an arbitrary real constant, and $\sigma_{\nu}(t)$ possesses not more than $n+1$ points of growth. Thus the formula (3.14) or the equivalent formula (3.15) give all canonical solutions of the truncated Hamburger moment problem in the non-degenerate case while the real parameter $a_{n}$ or the real parameter $\nu$, respectively, run through the real axis. 
To obtain the formula for $\Omega(z)$ in the degenerate case without loss of generality we can assume that

$$
\operatorname{rank} \Lambda_{n}=n \text {. }
$$

Otherwise we might ignore some last moments and consider a Hankel matrix $\Lambda_{n^{\prime}}=\left(\mu_{j+k}\right)_{j, k=0}^{n^{\prime}}$ with $n^{\prime}<n$, for which

$$
\operatorname{rank} \Lambda_{n^{\prime}}=n^{\prime} .
$$

By (3.16) there is only one ( up to a multiple ) non-zero vector $\boldsymbol{\xi}_{0}$ such that $\Lambda_{n} \boldsymbol{\xi}_{0}=0$ and condition 2 of Theorem 2.1 together with (3.16) provide that for this vector $\xi_{n} \neq 0$. As follows, for this vector the corresponding polynomial $d(t) \equiv P_{0}(t)$ has exactly degree $n$. In the space of polynomials $\mathfrak{P}_{n}$ of degree not more than $n$ corresponding to vectors of the space $\mathbb{C}^{n+1}$ the polynomials corresponding the one-dimensional subspace $\mathcal{L}$ form the one-dimensional space $\{\alpha d(t)\}$, where $\alpha$ runs the set of complex numbers $\mathbb{C}$. Classes of polynomials corresponding to elements $\mathbf{f}$ of the factors space $\mathcal{F}=\mathbb{C}^{n+1} \backslash \mathcal{L}$ from $\mathfrak{P}_{n}$ can be represented in the form $f(t)+\{\alpha d(t)\}$, where $f(t)$ is a polynomial of degree not more than $n-1$. Consider for $z, \operatorname{Im} z>0$, and an arbitrary $\mathbf{f} \in \mathcal{F}$ the equation

$$
(\mathrm{B}-z) \mathbf{g}=\mathbf{f}
$$

where B is the self-adjoint operator in $\mathcal{F}$ defined by (2.18). For the polynomial $g(t)$ of degree $\leq n-1$ representing the class of polynomials corresponding to $\mathbf{g},(3.17)$ transforms into equation

$$
(t-z) g(t)=f(t)+\alpha d(t)
$$

where $f(t)$ is the polynomial of degree $\leq n-1$ representing f. For (3.18) would give as the solution a polynomial of degree $\leq n-1$ the coefficient $\alpha$ in the right hand side of (3.18) must be equal to

$$
-\frac{f(z)}{d(z)}
$$

As in the proof of Proposition 2.2 we can verify that the suggestion that $d(z)$ has complex leads to the absurd conclusion that the self-adjoint operator B has complex eigenvalues. Therefore,

$$
g(t)=\frac{f(t) d(z)-f(z) d(t)}{(t-z) d(z)} .
$$

In particular, for the case $\mathbf{f}=\tilde{\mathbf{e}}_{0}$ the corresponding representing polynomial of degree $\leq n-1$ is $e_{0}(t) \equiv 1$ we obtain that

$$
g(t)=\frac{1}{d(z)} \frac{d(z)-d(t)}{t-z} .
$$


Setting

$$
e(z)=\int_{-\infty}^{\infty} \frac{d(t)-d(z)}{t-z} d \sigma(t)
$$

where $d \sigma(t)$ is the unique solution of the truncated Hamburger moment problem for the degenerate case, we conclude that $d \sigma(t)$ can be calculated immediately by poles and corresponding residues at them of the rational function

$$
\Omega(z)=\left\langle\left\langle(\mathrm{B}-z)^{-1} \tilde{\mathbf{e}}_{0}, \tilde{\mathbf{e}}_{0}\right\rangle\right\rangle=\int_{-\infty}^{\infty} \frac{d \sigma(t)}{t-z}=\frac{e(z)}{d(z)}
$$

\section{Non-canonical solutions of Hamburger's problem: Nevanlinna's theorem}

Hereafter the matrix $\Lambda_{n}$ is considered to be strictly positive. In this case formula (3.15) evidentlyestablishes a one-to one correspondence between the set of all real numbers and the subset of all canonical solutions of the truncated Hamburger moment problem. In this section applying as above the operator approach to the moment problem we show that (3.15) is a particular case of the general Nevanlinna formula giving description of both canonical and non-canonical solutions of that problem.

To this end note that each self-adjoint extension $\mathrm{A}_{\text {of }} \mathrm{A}_{0}$ in the space $\mathcal{H}$ or a defined on a larger self-adjoint extension of $\mathrm{A}_{0}$ Hilbert space $\mathcal{G}$ generates by formulas (3.1), (3.2) some solution $\sigma(t)$ of the truncated Hamburger problem. On the other hand it follows from the above constructions that each solution $\sigma(t)$ of this problem generates some self-adjoint extension A of $\mathrm{A}_{0}$, which is unitary equivalent to the natural extension of the symmetric operator of multiplication by the independent variable $t$ from the subspace $\mathfrak{P}_{n}$ of polynomials of degree $\leq n$ in the standardly defined space $\mathbf{L}_{\sigma}^{2}$ to the multiplication operator on $t$ in the whole space $\mathbf{L}_{\sigma}^{2}$. To obtain the complete description of all solutions of the truncated Hamburger problem starting from (3.1), (3.2) it is natural to use the M.G. Krein formula for the generalized resolvent [7], [3].

Let $\mathrm{A}_{1}$ be some self-adjoint extension of $\mathrm{A}_{0}$ in $\mathcal{H}$ and let $\widehat{\mathrm{A}}$ be an arbitrary self-adjoint extension of $\mathrm{A}_{0}$ acting in the Hilbert space $\mathcal{G}, \mathcal{H} \subseteq \mathcal{G}$. We keep for the extension of the scalar product of the space $\mathcal{H}$ onto the space $\mathcal{G}$ the same symbol $\langle\cdot, \cdot\rangle$. Note that in our special case $A_{0}$ and $A_{1}$ as operators acting in a finite dimensional space are bounded and the vector $\mathbf{h}_{n} \in \mathcal{H}$ introduced above is orthogonal to the domain of the symmetric operator 
$\mathrm{A}_{0}$. Then for arbitrary $\mathbf{f}, \mathbf{g} \in \mathcal{H}$ and any $z, \operatorname{Im} z>0$, the M.G. Krein formula is valid

$$
\begin{aligned}
\left\langle(\widehat{A}-z)^{-1} \mathbf{f}, \mathbf{g}\right\rangle & =\left\langle\left(\mathrm{A}_{1}-z\right)^{-1} \mathbf{f}, \mathbf{g}\right\rangle \\
& -\frac{\left\langle\left(\mathrm{A}_{1}-z\right)^{-1} \mathbf{f}, \mathbf{h}_{n}\right\rangle\left\langle\left(\mathrm{A}_{1}-z\right)^{-1} \mathbf{h}_{n}, \mathbf{g}\right\rangle}{\left\langle\left(\mathrm{A}_{1}-z\right)^{-1} \mathbf{h}_{n}, \mathbf{h}_{n}\right\rangle+\tau(z)},
\end{aligned}
$$

where $\tau(z)$ is some Nevanlinna function depending only on the extensions $\widehat{\mathrm{A}}, \mathrm{A}_{1}$ but not on the choice of vectors $\mathbf{f}, \mathbf{g} \in \mathcal{H}$. The M.G. Krein formula (4.1) establishes the one-to-one correspondence between the set of generalized resolvents of all self-adjoint extensions of the symmetric operator $\mathrm{A}_{0}$ and the set of all Nevanlinna functions, holomorphic in the upper half-plane.

By the connection between solutions of the truncated Hamburger moment problem and self-adjoint extensions of $\mathrm{A}_{0}$ and the M.G. Krein formula (4.1), the set of functions

$$
\Omega(z)=\int_{-\infty}^{\infty} \frac{d \sigma(t)}{t-z},
$$

where non-decreasing functions $\sigma(t)$ run through the set of all solutions of the truncated Hamburger moment problem, coinside with the set of functions

$$
\begin{aligned}
\Omega(z) & =\left\langle\left(\mathrm{A}_{1}-z\right)^{-1} \mathbf{e}_{0}, \mathbf{e}_{0}\right\rangle \\
& -\frac{\left\langle\left(\mathrm{A}_{1}-z\right)^{-1} \mathbf{e}_{0}, \mathbf{h}_{n}\right\rangle\left\langle\left(\mathrm{A}_{1}-z\right)^{-1} \mathbf{h}_{n}, \mathbf{e}_{0}\right\rangle}{\left\langle\left(\mathrm{A}_{1}-z\right)^{-1} \mathbf{h}_{n}, \mathbf{h}_{n}\right\rangle+\tau(z)},
\end{aligned}
$$

where $\tau(z)$ runs through the set of all Nevanlinna functions in the upper half-plane. Rather simple calculations using explicit representations (3.11), $(3.12)$ of $\left(\mathrm{A}_{1}-z\right)^{-1}$ in the space of polynomials $\mathfrak{P}_{\mathfrak{n}}$ yield in addition to $(3.14)$

$$
\begin{aligned}
\left\langle\left(\mathrm{A}_{1}-z\right)^{-1} \mathbf{e}_{0}, \mathbf{h}_{n}\right\rangle & =\left[\left(a_{n 1}-z\right) h_{n}(z)+b_{n-1} h_{n-1}(z)\right]^{-1}, \\
\left\langle\left(\mathrm{~A}_{1}-z\right)^{-1} \mathbf{h}_{n}, \mathbf{h}_{n}\right\rangle & =\left[\left(a_{n 1}-z\right) h_{n}(z)+b_{n-1} h_{n-1}(z)\right]^{-1} h_{n}(z),
\end{aligned}
$$

where $a_{n 1}$ is the parameter in (2.12) for the canonical extension $\mathrm{A}_{1}$. Further elementary calculations with account of the last relations results in the following formula

$$
\begin{aligned}
\Omega_{w}(z) & =\int_{-\infty}^{\infty} \frac{1}{t-z} d \boldsymbol{\sigma}_{w}(t)=-\frac{(w(z)-z) k_{n}(z)+b_{n-1} k_{n-1}(z)}{(w(z)-z) h_{n}(z)+b_{n-1} h_{n-1}(z)} \\
w(z) & =a_{n 1}+\frac{1}{\tau(z)} .
\end{aligned}
$$


Evidently $w(z)(4.5)$ is a Nevanlinna function in the upper half-plane. By construction $-\Omega_{w}(z)$ is also such function. Taking arbitrary canonical solution $d \sigma_{\nu}(t)$ consider the difference between the corresponding function $\Omega_{\nu}(z)$ and $\Omega_{w}(z)$. Applying the Christoffel identity for the orthogonal polynomials

$$
k_{n}(z) h_{n-1}(z)-k_{n-1}(z) h_{n}(z) \equiv \frac{\Delta_{n-1}}{\sqrt{\Delta_{n} \Delta_{n-2}}}
$$

we see that

$$
\begin{aligned}
& \Omega_{w}(z)-\Omega_{v}(z) \\
& =\frac{\nu-w(z)}{\left((w(z)-z) h_{n}(z)+b_{n-1} h_{n-1}(z)\right)\left((\nu-z) h_{n}(z)+b_{n-1} h_{n-1}(z)\right)} .
\end{aligned}
$$

Recall that for the canonical solutions the asymptotic relation (3.3) holds. By (4.6) the relation

$$
\left|\Omega_{w}(z)-\Omega_{v}(z)\right|=o\left(\frac{1}{|z|^{2 n+1}}\right)
$$

holds for $z \rightarrow \infty$ uniformly in any angle $\delta \leq \arg (z) \leq \pi-\delta, 0<\delta<\pi$ if and only if

$$
\lim _{z \rightarrow \infty} \frac{w(z)}{z}=0
$$

uniformly inside any such angle. Hence for $\Omega_{w}(z)$ the asymptotic relation holds if and only if $w(z)$ satisfies the condition (4.7). Denote by $\mathbb{N}_{0}$ the subset of Nevanlinna functions in the upper half-plane, which satisfy the condition (4.7). The obtained results can be summarized in the following theorem.

Theorem 4.1. The Nevanlinna formula

$$
\begin{aligned}
\int_{-\infty}^{\infty} \frac{1}{z-t} d \boldsymbol{\sigma}_{w}(t) & =\frac{b_{n-1} k_{n-1}(z)-(z+w(z)) k_{n}(z)}{b_{n-1} h_{n-1}(z)-(z+w(z)) h_{n}(z)} \\
& =\frac{k_{n+1}(z)+\widetilde{w}(z) k_{n}(z)}{h_{n+1}(z)+\widetilde{w}(z) h_{n}(z)} \\
\widetilde{w}(z) & =\frac{\hat{a}_{n}+w(z)}{b_{n}}
\end{aligned}
$$

establishes in the non-degenerate case the one-to-one correspondence between the set of Nevanlinna functions $w(z) \in \mathbb{N}_{0}$ and the set of all solutions of the truncated Hamburger moment problem. 
Acknowledgment. We are grateful to V. M. Adamjan for his valuable aid. The authors also thank the referee for the suggestions which lead to a significant improvement of the text.

\section{References}

[1] Adamyan, V. M. and Tkachenko, I. M., High-frequency electrical conductivity of a collisional plasma, High Temp. (USA) 21, (1983), 307.

[2] Akhiezer, N. I., The Classical Moment Problem and Some Related Questions in Analysis, Hafner Publishing Company, New York, 1965.

[3] Akhiezer, N. I. and Glazman, I. M., Theory of Linear Operators in Hilbert Space, Frederick Ungar Publishing Company, New York, 1963, Vol II, § 59.

[4] Curto, R. E. and Fialkow, L. A., Recursiveness, positivity, and truncated moment problems, Houston J. Math. 17 (1991), 603; see also: Curto, R. E. and Fialkow, L. A., Solutions of the Truncated Moment Problem, Mem. Amer. Math. Soc. 119 (1996).

[5] Hamburger, H., Über eine Erweiterung des Stieltjesschen Momentenproblems, Math. Ann. 81 (1920), 235; ibid, 82 (1921), 120; ibid, 82 (1921), 168.

[6] Jones, W. B., Njåstad, O., Thron, W. J., Moment theory, orthogonal polynomials, quadrature, and continued fractions associated with the unit circle, Bull. London Math. Soc. 21 (1989), 113-152.

[7] Krein, M. G., The theory of extensions of semibounded Hermitian operators and its applications (in Russian), Mat. Sb. 20 (1947), 431-495.

[8] M.G. Krein, The description of all solutions of the truncated power moment problem and some problems of operator theory, Mat. Issled. 2(2) (1967), 114-132; English translation, Amer. Math. Soc. Transl. Ser. 295 (1970), 219-234.

[9] Krein, M. G., Nudel'man, A. A., The Markov Moment Problem and Extremal Problems, Transl. Math. Monographs 50 (1977), Amer. Math. Soc., Providence, RI.

[10] Landau, H. J. (Ed.), Moments in Mathematics, Proc. Sympos. Appl. Math. 37 (1987), Amer. Math. Soc., Providence, RI.

[11] Ortner, J., Rylyuk, V. M., Tkachenko, I. M., Reflectivity of cold magnetized plasmas, Phys. Rev. E 50 (1994), 4937.

[12] Shohat, J. A., Tamarkin, J. D., The Problem of Moments, Math. Surveys Monographs 1 (1943), Amer. Math. Soc., Providence, RI, (4 ${ }^{\text {th }}$ Ed. 1970).

[13] Stieltjes, T. J., Recherches sur les fractions continues, Ann. Fac. Sci. Toulouse Math. 8 (1894), J76-J122; ibid, 9 (1895), A5-A47.

M. URREA

DEPARTMENT OF APPLIED

MATHEMATICS

ETSII, Polytechnic University

E-46071 VALENCIA

SPAIN

EMAIL: MURREA@MAT.UPV.ES
I. M. TKACHENKo

Department of ApPlied

Mathematics

ETSiI, Polytechnic University

E-46071 VALENCIA

SPAIN 
P. Fernández de Córdoba

Department of Applied Mathematics

ETSII, Polytechnic University

E-46071 VALENCIA

SPAIN 